MATEC Web of Conferences 25,03004

(2015)

DOI: $10.1051 /$ matecconf/ 20152503004

(C) Owned by the authors, published by EDP Sciences, 2015

\title{
Research on Underwater Vehicle Based on Multiphase Flow Control
}

\author{
Xiaoshi Zhang \& Cong Wang \\ School of Astronautics, Harbin Institute of Technology, Harbin, Heilongjiang, China
}

\begin{abstract}
The commercial software ANSYS CFX is used for modeling the hydrodynamic characteristics of submarine-launched vehicle. In the numerical simulations, the SST (Shear Stress Transport) turbulence model is employed to describe the turbulence phenomenon occurring in the continuous fluid. The Rayleigh-Plesset equations are applied to analyze the cavitation phenomenon. Three-dimensional numerical simulation was carried out to study the cavity shape, the surface pressure distribution and the drag force with different flow control. The result shows that the gas and the number of ventilation holes control to show any significant impact on the cavity shape and the surface pressure are effective measures to decrease resistance.
\end{abstract}

Keywords: flow controlling; ventilation holes; cavity shape; multiphase flow; numerical simulation

\section{INTRODUCTION}

The current work is motivated by recent efforts to develop high-speed underwater vehicles. Cavitation plays an important role in the development of such vehicles because of the unsteady features associated with this type of flow. It causes unsteady hydrodynamic forces, severe noise, structural erosion, and vibration problems. These cavities tend to collapse due to the reentrant of a water jet and the flow becomes unsteady, which has significant effects on the stability and the trajectory of the vehicle. In order to control these problems, active control is adapted. Active control can reduce the drag and keep cavity stability. As to this thesis, many scholars have did much work and issued their own conclusions and gained their research results. Active control can be divided into the thruster vector control, the grid fin control, and the ventilation method. Ding ${ }^{[1]}$ established the mathematical physical models of thrust vector control systems for underwater vertical launching trajectories. The thrust vector can ensure missile exit in the given attitude, and the control trajectories have good dynamic character. Hydrodynamic characteristic of grid fin for missile applications has been numerically studied. Calculated results indicate that the grid fin using as a hydrodynamic stabilizers and control surfaces has some good performances in several aspects. Cavitation might reduce the lift-drag ratio, as well as the efficiency of grid fin ${ }^{[2]}$. A test was carried out to investigate the influences on the frame profile of grid fin and the number of grid plate on the hydrodynamic characteristics of grid fin. The drag and lift force will increase with rising the number of grid plate. The lift force and the drag will be reduced when producing cavitation. The simulation has been adapted to obtain the rules of normal force, axial force, hinge moment coefficient changed with deflection angle ${ }^{[4]}$. A study has been carried out to determine various aspects of the flow physics of a supercavitating vehicle. Results regarding supercavity shape, closure, and ventilation requirements versus Froude number are presented ${ }^{[7]}$ Experimental and modelling studies of air cavities formed under a model-scale hull are reported. The application of air-ventilated cavities for marine vessels can result in substantial drag reduction ${ }^{[8]}$. The unsteady characteristics of ventilated cavitating flows around axisymmetric body with a cavitator are addressed by using experimental methods. Cavities around the body are observed by means of a high-speed video camera in the different conditions ${ }^{[9]}$. The cavitation flow characteristics and hydrodynamic evolution during the rise of underwater launched missile were researched by using a numerical simulation method $^{[10]}$.

The paper is organized as follows. The description of the physical problem is firstly presented, and this is followed by the computational methods. The computational results are then presented and discussed. Finally, a summary and the conclusions are provided.

\section{NUMERICAL MODEL}

\subsection{Governing equations}

The continuity equation:

$$
\frac{\partial \rho_{n}}{\partial t}+\frac{\partial}{\partial x_{j}}\left(\rho_{n} u_{j}\right)=0
$$

The momentum equation:

$$
\begin{aligned}
& \frac{\partial}{\partial t}\left(\rho_{n} u_{i}\right)+\frac{\partial}{\partial x_{j}}\left(\rho_{n} u_{i} u_{j}\right)=-\frac{\partial p}{\partial x_{i}}+ \\
& \frac{\partial}{\partial x_{j}}\left[\left(\mu_{n}+\mu_{t}\right)\left(\frac{\partial u_{i}}{\partial x_{j}}+\frac{\partial u_{j}}{\partial x_{i}}\right)\right]
\end{aligned}
$$

*Corresponding author: hitzxs@gmail.com

This is an Open Access article distributed under the terms of the Creative Commons Attribution License 4.0, which permits unrestricted use, distribution, and reproduction in any medium, provided the original work is properly cited. 


\section{MATEC Web of Conferences}

Where $u_{j}$ is the velocity; $i$ and $j$ denote the coordinate direction; $\alpha_{1}, \alpha_{2}, \alpha_{3}$ are respectively liquid, gas and vapor; is the mixture $\rho_{n}$ e density; is the mixture dynamic viscosity.

\subsection{Cavitation model}

The Shear Stress Transport (SST) turbulence model is used in this paper, which solves the transport problem of the turbulence shear force by adding the eddy viscosity limited equation to the Baseline $\mathrm{k}-\omega$ turbulence model, and it can perform more accurate prediction for the flow separation problem under the condition of adverse pressure gradient. The baseline turbulence model is shown as follows:

$$
\begin{aligned}
& \frac{\partial}{\partial t}\left(\rho_{n} k\right)+\frac{\partial}{\partial x_{j}}\left(\rho_{n} u_{j} k\right)= \\
& \frac{\partial}{\partial x_{j}}\left[\frac{\partial k}{\partial x_{j}}\left(\mu+\frac{\mu_{t}}{\sigma_{k 3}}\right)\right]+G_{k}+G_{b}-\beta^{\prime} \rho k \omega \\
& \frac{\partial\left(\rho_{n} \omega\right)}{\partial t}+\frac{\partial}{\partial x_{j}}\left(\rho_{n} u_{j} \omega\right)=\frac{\partial}{\partial x_{j}}\left[\left(\mu+\frac{\mu_{t}}{\sigma_{\omega 3}}\right) \frac{\partial \varepsilon}{\partial x_{j}}\right]+ \\
& \alpha_{3} \frac{\omega}{k} P_{k}+P_{\omega b}+2\left(1-Y_{1}\right) \rho \frac{1}{\sigma_{\omega 2} \omega} \frac{\partial k}{\partial x_{j}} \frac{\partial \omega}{\partial x_{j}}-\beta_{3} \rho \omega
\end{aligned}
$$

The eddy viscosity limited equation is added to the SST turbulence model:

$$
v_{t}=\frac{b_{1} k}{\max \left(b_{1} \omega, \Omega Y_{2}\right)}
$$

Where $\mu_{t}$ is the turbulence viscosity; $Y_{1}$ and $Y_{2}$ is the mixture function; $G_{k}$ is the turbulent kinetic energy generation formula caused by the viscosity force; $G_{b}$ is the turbulent kinetic energy generation formula caused by $\mu_{n}$ the buoyancy; $\Omega$ is the invariant measure under the strain rate.

\subsection{Cavitation model}

P A transport equation model including gas and vapor mass fraction equation was employed to simulate cavitation, the equation is expressed as follows:

$\frac{\partial}{\partial t}\left(\alpha_{i} \rho_{i}\right)+\nabla \cdot\left(\alpha_{i} \rho_{i} U_{i}\right)=\Psi_{i}+\Gamma_{i}$

The source terms and $\Gamma_{i}$ respectively represent the vaporization and condensation.

The single bubble dynamics can be modeled through the simplified Rayleigh-Plesset equation, neglecting the second order term and the surface tension, as shown below:

$\frac{d R_{g}}{d t}= \pm \sqrt{\frac{2\left(p_{c}-p\right)}{3 \rho}}$

Where $R_{g}$ is the bubble radius; $p_{c}$ is the vapor pressure; $p$ is the flow surrounding.

The volume fraction is related to the bubble number density $N_{g}$ and the bubble radius in the cavitation structure

$\gamma_{b}=V_{g} N_{g}=4 / 3 \pi R_{g}^{3} N_{g}$

\section{GEOMETRY AND BOUNDARY CONDITIONS}

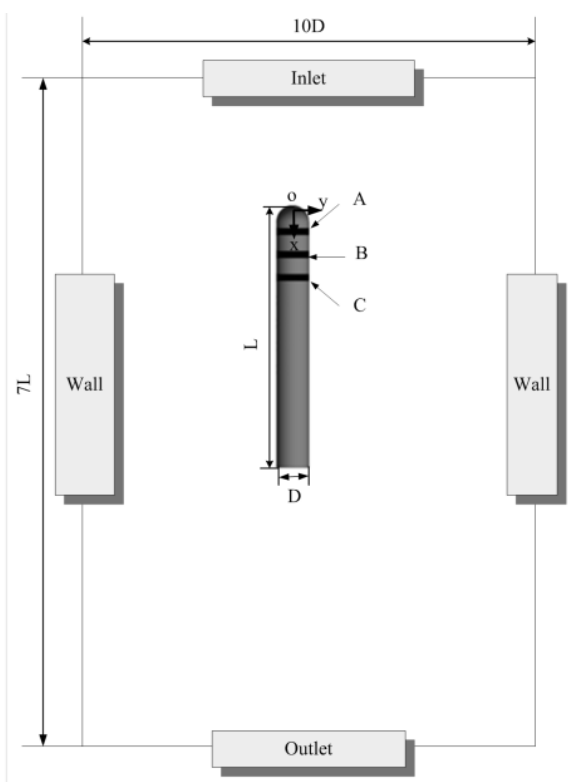

Figure 1. Computational geometry model and boundary

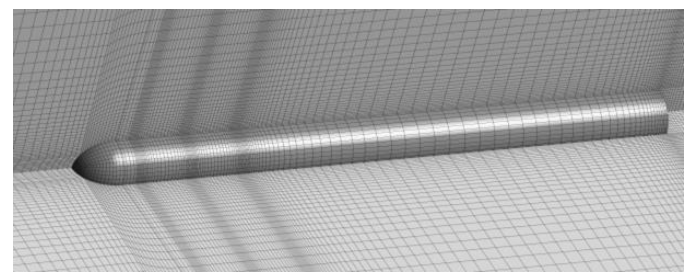

Figure 2. Mesh of model

An O-type, structured computational grid has been utilized to simulate an underwater vehicle which is fixed in the middle of the domain vertically. The simulation is performed in three dimensions and for promoting the precision, the grid is more concentrated near the vehicle. The vehicle has diameter $\mathrm{D}$ and length $\mathrm{L}$. The left and right boundaries are located at 5D from the center of the vehicle. The boundaries of upstream and the downstream are located at $2 \mathrm{~L}$ and $5 \mathrm{~L}$. The schematic configuration of the computational domain is shown in Figure 1. The boundary condition is set on inlet, outlet and wall. The velocity is fixed at the domain inlet and the pressure opening is imposed at the outlet. The mass inlet boundary is defined at the ventilation holes. The no-slip wall conditions are set 
for the lateral boundaries and the body. After a grid independency solution study, an 800000 mesh is used for all computations around the vehicle. Figure 2 shows the mesh structure in the computational domain. The ventilation holes are named A, B and C. The ventilation angle is shown in Figure 1.

\section{NUMERICAL RESULTS}

\subsection{Verification by experiment}

By applying this method to Cavitation flow fields around axisymmetric revolution bodies, the validity and the feasibility of this method were verified.

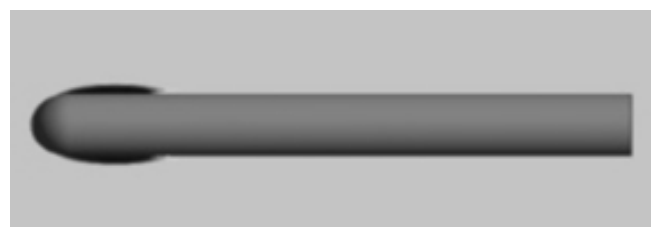

Figure 3. Contour at liquid volume fraction $\alpha$

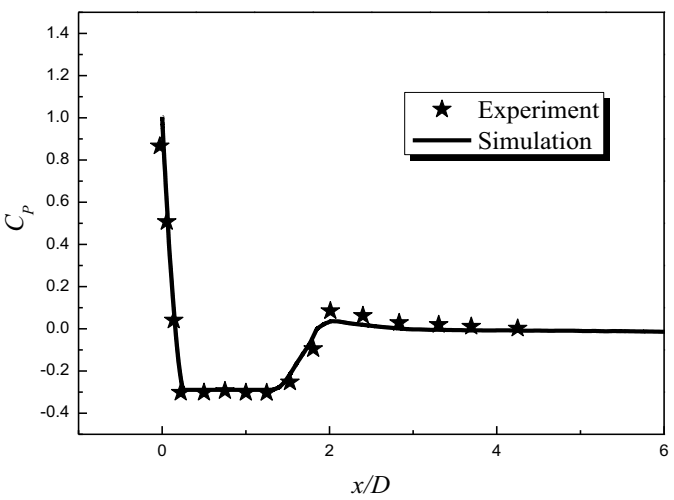

Figure 4. Comparison of numerical results and experiment data

Volume fraction was introduced to distinguish water and vapor phases as shown in

Figure 3. As shown in 错误!未找到引用源。, the pressure distribution on the hemispherical head-form body surface for cavitation number 0.3 was compared with existing experimental data. The computed pressure distribution in the flows showed good agreement with the existing experimental data.

\subsection{Pressure distribution and surface pressure}

The numerical investigation is carried out with different number of ventilation holes. In the process of computation, the vehicle is perpendicular to the water and the vehicle head is the origin of coordinate system. The local pressure $p_{0}$ of the origin is $3129 \mathrm{~Pa}$ and $p_{0}+\rho g x$ in the direction of the positive axis $\mathrm{x}$. The value of vapor pressures and reference pressure is respectively $3510 \mathrm{~Pa}$ and $101325 \mathrm{~Pa}$.

Table 1 shows the positions of different ventilation holes. Figure 5 shows cavity shapes at different numbers of ventilation holes. At the same ventilation velocity, cavity shapes and the tail cavity vary at different numbers of ventilation holes and third model forms the closure tail cavity.

Table 1. Positions of ventilation holes.



Figure 5. Cavity shapes at different number of ventilation holes

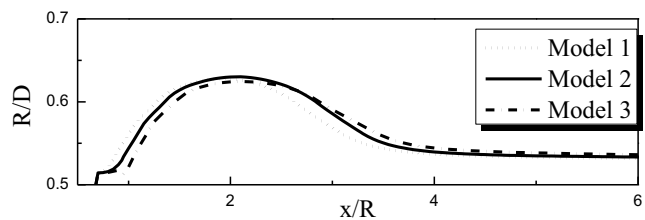

(a) $\sigma=0.25$

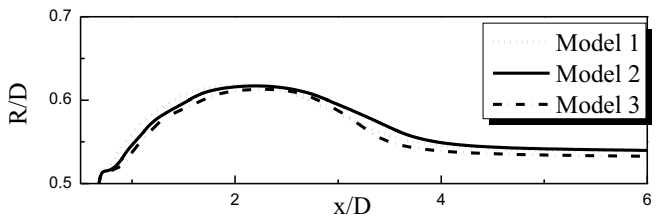

(b) $\sigma=0.30$



(c) $\sigma=0.35$

Figure 6. Cavity radius of different models 


\section{MATEC Web of Conferences}

Figure 6Figure 7 shows the numerical cavity radius of different models at the same cavitation number.

Model 1 has the maximum radius before $x / D=2$ and the minimum radius after $x / D=2$ as shown in Figure 6(a). The radius of different models has similar variation trend with different cavitation numbers.

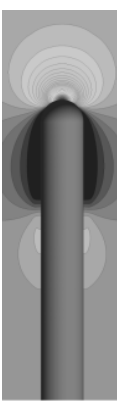

(a)

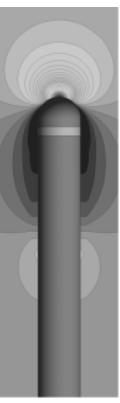

(b)

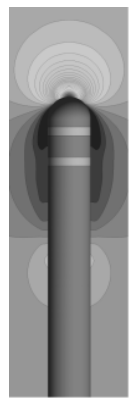

(c)

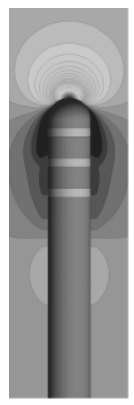

(d)
Figure 7. Comparison of pressure contours in the case of different number of ventilation holes

Figure 7 shows the numerical surface pressure distributions at cavitation number 0.25 . Figure 7(a) shows pressure contours of the natural cavitating flows. A comparison among these four contours concludes cavitation that can be controlled by ventilation. With the number of ventilation holes increasing, the pressure will be different.

Figure 8 shows the surface pressure coefficient of different models at the same cavitation number. $\mathrm{Cp}$ is the surface pressure coefficient. The increment of cavitation number is from 0.25 to 0.3 , pressure peak is firstly found in model 1 at $x / D=0.75$. Pressure peaks are found in model 2 and model 3 as the increasing cavitation number from 0.25 to 0.3 and the pressure peak of model 1 continues to become higher. At the same cavitation number, the pressure peaks of model 1 and model 3 are respectively the maximum and minimum.

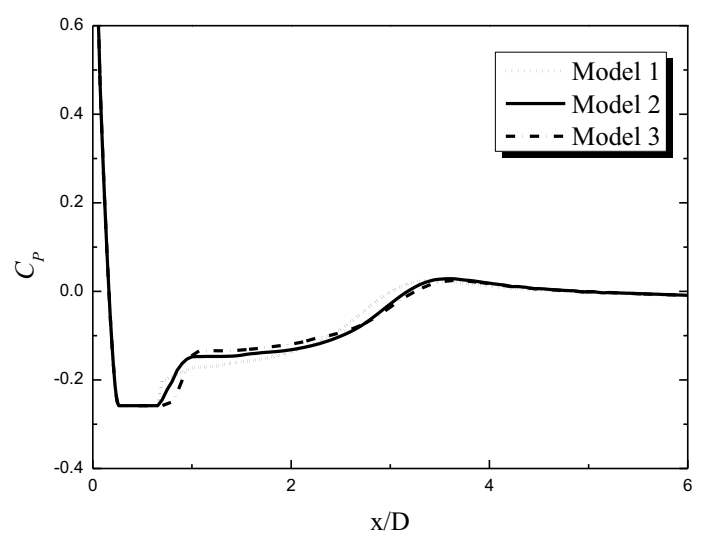

(a) $\sigma=0.25$

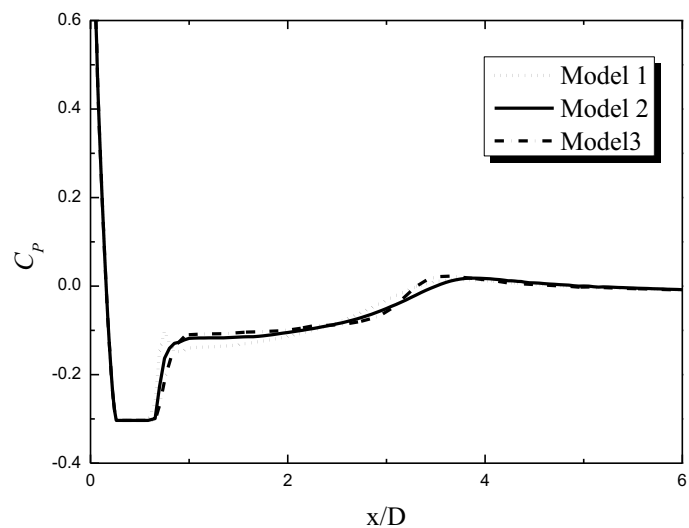

(b) $\sigma=0.30$

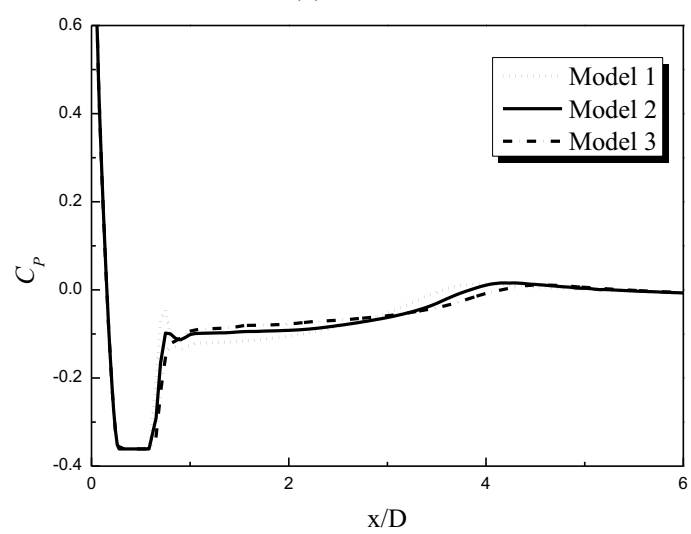

(c) $\sigma=0.35$

Figure 8. Surface pressure coefficient of different model

\subsection{Analysis of drag force}

In this calculation, the drag coefficients of different models were analyzed and compared with other ventilation rate.



(a) Comparison of pressure force coefficient at different mass flow 


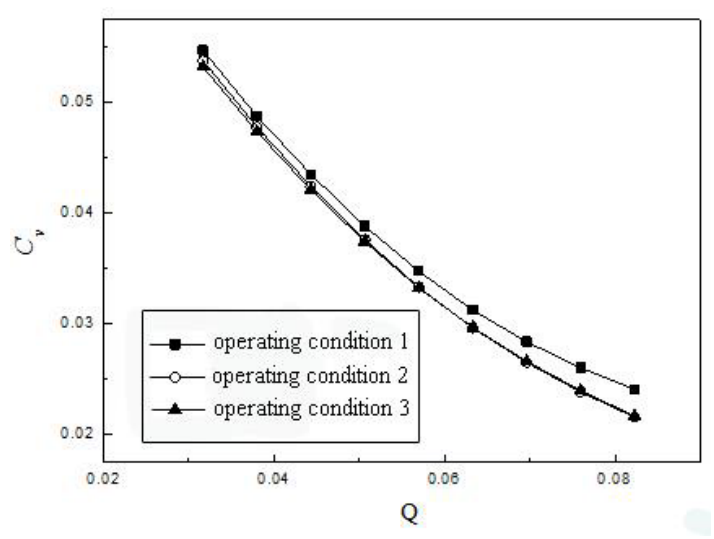

(b) Comparison of viscous drag coefficient at different mass flow

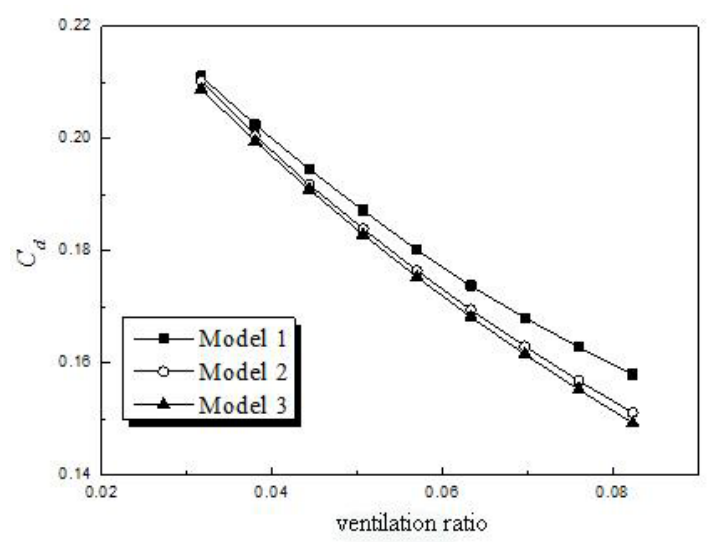

(c) Comparison of total drag coefficient at different mass flow

Figure 9. Comparison of drag coefficient force coefficient in the case of different numbers of ventilation holes

Figure 9 shows the drag coefficient force coefficient. $C_{w}$ is the pressure force coefficient; $C_{v}$ is the viscous drag coefficient; $C_{d}$ is the total coefficient; $Q$ is the ventilation rate.

At the same ventilation rate, the pressure force coefficient and viscous drag coefficient decrease with the increasing number of ventilation hole and the total coefficient decrease. At the same ventilation rate, the more the number of ventilation holes is, the faster the pressure force coefficient will decrease; the viscous coefficients of the second model and the third model are smaller than the first one. The cavity shape of the three models is similar. The third model has the smallest pressure force coefficient. The total pressure coefficient includes the pressure coefficient and the viscous drag coefficient, so the total pressure coefficient decreases with the increasing number of ventilation hole; the more the ventilation hole is, the total pressure coefficient decreases faster with the increas- ing ventilation rate. At the same condition, the number of ventilation holes and ventilation rate has an even greater impact on the drag force to show a reasonable design and to obtain the best drag reduction effect.

\section{CONCLUSION}

In this paper, the three-dimensional numerical simulation method is adopted to the analysis about the cavity shape, surface pressure coefficient and drag coefficient at different numbers of ventilation holes:

(1)At the same ventilation rate, the cavity length increases and forms the closure tail cavity with the increasing number of ventilation hole

(2)There is small natural cavitation field under the ventilation condition. The pressure peak exists in the ventilation holes and it increases with the increasing cavitation.

(3)In different models, the pressure force coefficient and the viscous drag coefficient decrease with the increasing ventilation rate. The more the ventilation holes are, the more obvious it decreases. The decrease of viscous drag coefficient becomes obvious as the number of ventilation hole increases and the trend of the total drag coefficient will decrease.

\section{REFERENCES}

[1] Ding, Y.C. \& Wang, B.S. 2011. Study on the thrust vector control trajectory of underwater vertical launching missile. Journal of Ship Mechanics, (Z1):87-94.

[2] Hang, T, Wu, L. \& Lu, C.J, et al. 2006. Cavitaing grid gin hydrodynamics for missile applications. Journal of hydrodynamics (A), (02):239-243.

[3] Xia, Y.Y, Chen, W.Q. \& Wang, B.S. 2014. Cavitation Hydro-dynamic Test for a New Grid Fin in Water Tunnel. Torpedo technology, (05):321-324+328.

[4] Yao, Y. \& Mao, H.Y. 2004. Numerical simulation of hydrody-namic characteristics for grid Fins, Tactical missile technology, (02):13-17.

[5] Reichardt,H. 1945. The laws of cavitation bubbles at axially symmetrical bodies in a flow: Kaiser Wilhelm Institute für Stromungsforschung.

[6] Sliberman, E. \& Song, C.S. 1959.Instability of ventilated cavities.

[7] Kawakami, E. \& Arndt, R.E. 2011.Investigation of the behavior of ventilated supercavities. Journal of Fluids Engineering, 133(9):091305.

[8] Matveev, K. \& Miller, M. 2011. Air cavity with variable length under a model hull. Proceedings of the Institution of Mechanical Engineers, Part M: Journal of Engineering for the Maritime Environment, 225(2):161-169.

[9] Wang, F.F, Wang, G.Y. \& Huang, B, et al. 2014. Experimental Study on Unsteady Ventilated Cavitating Flows around an Axisymmetric Body with Cavitator. Aca Armamentarii, (03):333-339.

[10]Min, J.X, Wei, Y.J. \& Wang, C, et al. 2010. Numerical simulation on hydrodynamic characteristics of submarine missile in the verticallaunch process. Aca Armamentarii, (10):1303-1309. 